\title{
THE EVOLUTION OF STARBURST GALAXIES TO ACTIVE GALACTIC NUCLEI
}

\author{
Nick Scoville \\ Owens Valley Radio Observatory \\ California Institute of Technology \\ Colin Norman \\ Space Telescope Science Institute and \\ Johns Hopkins University
}

\begin{abstract}
Recent observations of luminous IRAS galaxies $\left(\mathrm{L}_{I R}>10^{11} \mathrm{~L}_{\odot}\right)$ have revealed that virtually all are extremely rich in molecular gas with $\mathrm{H}_{2}$ abundances 5-20 times that of the Milky Way, and deep CCD imaging indicates that most are recent galactic mergers. Interferometric observations at millimeter wavelengths for five of these galaxies demonstrate that approximately half of the interstellar matter is contained in the central kpc. This gas concentration can result in the formation of a massive central star cluster. The deep potential of the central star cluster and the high density of interstellar gas ensure that virtually all of the gas lost during late stellar evolution sinks to the center of the cluster, building up a central, massive black hole. For a coeval star cluster of $4 \times 10^{9} \mathrm{M}_{\odot}$, a central mass of approximately $1.5 \times 10^{9} \mathrm{M}_{\odot}$ will accumulate within approximately $10^{8}$ years and accretion at an average rate of $7 \mathrm{M}_{\odot} \mathrm{yr}^{-1}$ over this time will result in a mean accretion luminosity of $10^{13} \mathrm{~L}_{\odot}$. This luminosity, radiated at $\mathrm{X}$-ray and uv wavelengths from the inner accretion disk ionizes the mass loss envelopes of the surrounding red giant stars providing an origin for the broad emission line regions of QSO's. The large linewidths would then be due to orbital motion in the massive central star cluster.
\end{abstract}

\section{Introduction}

Virtually all the luminous IRAS galaxies have also been shown to be extremely rich in interstellar gas, predominantly molecular hydrogen; this gas is also highly concentrated in their nuclei. Over the last year, the millimeter wave interferometer at Owens Valley Radio Observatory has been used for aperture synthesis mapping of the CO emission in five of the luminous galaxies (Scoville et al 1986, Sargent et al 1987, and Sanders et al 1987). In each case, 30-70\% of the total $\mathrm{CO}$ emission is confined to a region $\leq 10^{\prime \prime}$ in size centered on one of the galactic nuclei. Since all of these galaxies exhibit optical morphology indicative of galactic 


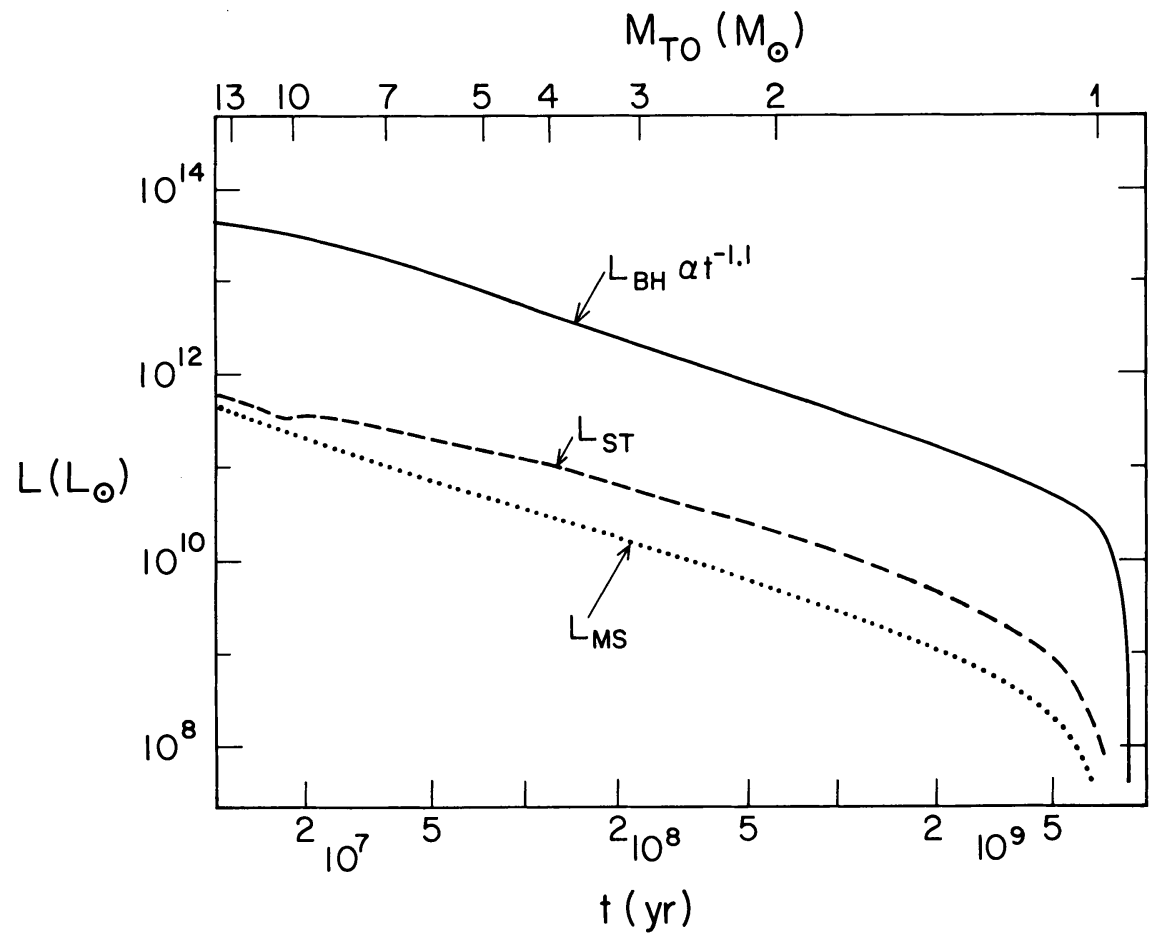

Fig. 1. The accretion luminosity, total stellar luminosity, and main sequence stellar luminosity are shown as a function of time for a coeval population of total mass $4 \times 10^{9} \mathrm{M}_{\odot}$ (Norman and Scoville 1988). At all times $<10^{9}$ years, the black hole accretion luminosity exceeds the total stellar luminosity by more than a factor of 25 and the accretion luminosity decays as $t^{-1.1}$.

mergers, it is apparent that the collision of two gas-rich galaxies can lead to the deposition of an enormous quantity of interstellar matter in the central few kpc. A high efficiency of star formation in this gas will then lead naturally to a massive central star cluster. We have recently undertaken a theoretical investigation of the evolution of such a system (Norman and Scoville 1988, Scoville and Norman 1988) and the results are briefly summarized below.

\section{Stellar Evolution in Nuclear Star Bursts}

Our model starts with the formation of a single coeval stellar cluster of total mass $4 \times 10^{9} \mathrm{M}_{\odot}$. This mass is distributed among stars with a Salpeter initial mass function $(\alpha=2.35)$ over the range $1-50 \mathrm{M}_{\odot}$. The total number of stars in the cluster is $1.4 \times 10^{9}$ and evolution of the stellar population is followed using the analytic work Renzini and Buzzoni (1986). The small radius of the cluster (10-50 pc) implies an extremely high escape velocity $\left(>10^{3} \mathrm{~km} \mathrm{~s}^{-1}\right)$. This fact together with a modest density of interstellar matter will ensure that all mass-loss occurring during the late stellar evolution phases (red giant mass-loss and supernovae) will be trapped in the cluster and eventually sink, dissipatively, to the center. Thus, 
from the stellar evolution calculations and the initial mass function, it is possible to predict the growth rate of the central black hole and its accretion luminosity.

In Figure 1, the black hole luminosity $\left(0.1 \dot{M} \mathrm{c}^{2}\right)$, the total stellar luminosity and the main sequence stellar luminosity are shown as a function of time. Over most of the time span $2 \times 10^{7}-5 \times 10^{9}$ years, all three luminosity components decay as power laws in time. The dominant luminosity contribution, provided by accretion onto the black hole, decays as $\mathrm{t}^{-1.1}$ (assuming a Salpeter IMF).

The bulk of the accretion luminosity will be radiated at uv and $\mathrm{x}$-ray wavelengths. This high flux of ionizing radiation will have immediate, observable effects on the mass-loss envelopes of red giant stars in the surrounding stellar cluster. For a star at distance $10^{18} \mathrm{~cm}$, the uv radiation will penetrate to a depth of $10^{14} \mathrm{~cm}$ and cause complete ionization in the zone outside this point. The structure of the mass-loss envelope will be that of an inverted Stromgren sphere with maximum electron density $10^{9} \mathrm{~cm}^{-3}$ and size $10^{14} \mathrm{~cm}$. These parameters are nearly identical to those derived for the broad emission line regions in QSOs, and Scoville and Norman (1988) suggest that the ionized mass-loss envelopes are the source of the broad line emission in AGNs.

Model emission line profiles were calculated for an ensemble of mass-loss stars distributed as a power law in distance from the central source. The high density transitions have line wings extending over $5000 \mathrm{~km} \mathrm{~s}^{-1}$ from line center and the profiles exhibit a logarithmic dependence on velocity in excellent agreement with the observed line profiles for QSOs (cf. Blumenthal and Mathews 1975). A noteworthy prediction of these calculations is the dependence of the line width on the critical density of the transition: with higher critical density transitions having greater velocity width due to the fact that stars closer to the central source are ionized deeper in the mass-loss envelope (where the density is higher) and these stars have higher orbital velocities. This is in agreement with the observational data for Seyfert galaxies (Filippenko et al. 1984).

\section{REFERENCES}

Blumenthal, G.R. and Mathews, W.G. 1975, Ap.J., 198, 517.

Filippenko, A.V., Huggins, P.J., Bregman, J.N., and Glassgold, A.E. 1984, Ap.J., 285, 458.

Norman, C.A. and Scoville, N.Z. 1988, Ap.J., 332, 124.

Renzini, A. and Buzzoni, A. 1986, in Spectral Evolution in Galaxies, ed. C. Chiosi and A. Renzini (Dordrecht: Reidel), p. 195.

Sanders, D.B., Scoville, N.Z., Sargent, A.I., and Soifer, B.T. 1987, Ap.J. (Letters), submitted.

Sargent, A.I., Sanders, D.B., Scoville, N.Z., and Soifer, B.T. 1987, Ap.J. (Letters), $312,235$.

Scoville, N.Z. and Norman, C.A. 1988, Ap.J., 332, 163.

Scoville, N.Z., Sanders, D.B., Sargent, A.I., Soifer, B.T., Scott, S.L., and Lo, K.Y. 1986, Ap. J. (Letters), 311, L47. 


\section{DISCUSSION}

PENSTON Although the overall line profiles may be 'logarithmic,' the wisps are in fact better fitted by a $1 / \Delta \lambda^{2}$ law. This can be explained by parabolically orbiting objects uniformly distributed in angular momentum which brighten as $R^{-2}$ as they approach the nucleus.

SCOVILLE We are modeling the logarithmic line profiles simply because that is a reasonable characterization of the observed profiles. Clearly, by combining different power laws for the stellar distribution, one can get almost any line shape, and clearly the observed line shapes are not the same in all objects. 